\title{
THE 1/2-COMPLEX BRUNO FUNCTION AND THE YOCCOZ FUNCTION. A NUMERICAL STUDY OF THE MARMI-MOUSSA-YOCCOZ CONJECTURE.
}

\author{
TIMOTEO CARLETTI
}

\begin{abstract}
We study the $1 / 2-$ Complex Bruno function and we produce an algorithm to evaluate it numerically, giving a characterization of the monoid $\hat{\mathcal{M}}=\mathcal{M}_{T} \cup \mathcal{M}_{S}$. We use this algorithm to test the Marmi-Moussa-Yoccoz Conjecture about the Hölder continuity of the function $z \mapsto-i \mathbf{B}(z)+\log U\left(e^{2 \pi i z}\right)$ on $\{z \in \mathbb{C}: \Im z \geq 0\}$, where $\mathbf{B}$ is the $1 / 2$-complex Bruno function and $U$ is the Yoccoz function. We give a positive answer to an explicit question of S. Marmi et al Marmi et al. 2001.
\end{abstract}

\section{INTRODUCTION}

The real Bruno functions are arithmetical functions $B_{\alpha}: \mathbb{R} \backslash \mathbb{Q} \rightarrow \mathbb{R}_{+} \cup\{+\infty\}$, $\alpha \in[1 / 2,1]$ which characterize numbers by their rate of approximation by rationals. They have been introduced by J.-C. Yoccoz Yoccoz 1995 (cases $\alpha=1 / 2$ and $\alpha=1$ ) and then studied in a more general context in Marmi et al. 1997.

For their relationship with arithmetical properties of real numbers, Bruno's functions enter in a huge number of dynamical system problems involving small divisors, for instance in the problem of the stability of a fixed point of a holomorphic diffeomorphism of a complex variable (the so called Schröder-Siegel problem) Yoccoz 1995, in the Schröder-Siegel problem in the Gevrey setting in one complex variable Carletti-Marmi 2000 and in some local conjugacy problems: Semistandard map [Marmi 1990, Davie 1994], analytic circle diffeomorphisms Yoccoz 2002] and some analytic area-preserving annulus map including the Standard map and some of its generalizations Berretti Gentile 2001.

Let us now concentrate on the $1 / 2$-Bruno function ${ }^{1} . B_{1 / 2}$ is $\mathbb{Z}$-periodic, even (for this reason it is also called even Bruno's function) and verifies the functional equation:

$$
B_{1 / 2}(x)=-\log x+x B_{1 / 2}\left(x^{-1}\right) \quad x \in(0,1 / 2) .
$$

The set $\mathcal{B}=\left\{x \in \mathbb{R}: B_{1 / 2}(x)<+\infty\right\}$ is called the set of Bruno's numbers: by (1.1) it follows that $\mathcal{B}$ is invariant under the action of the modular group $G L(2, \mathbb{Z})=$ $\left\{\left(\begin{array}{ll}a & b \\ c & d\end{array}\right): a, b, c, d \in \mathbb{Z}, a d-b c= \pm 1\right\}$. The Bruno function can be extended to rational numbers by setting $B_{1 / 2}(x)=+\infty$ when $x \in \mathbb{Q}$.

Date: November 10, 2018.

Key words and phrases. Complex Bruno Function, Yoccoz Function, linearization of quadratic polynomial, Littlewood Paley dyadic decomposition, continued fraction, Farey series.

${ }^{1}$ From Marmi et al. 1997 we know that the difference of any two Bruno's functions is in $L^{\infty}(\mathbb{R})$. 
Using the continued fraction algorithm one can solve (1.1) to obtain:

$$
B_{1 / 2}(x)=\sum_{k \geq 0} \beta_{k-1}(x) \log x_{k}^{-1},
$$

where $x_{0}=x, x_{k}=A_{1 / 2}\left(x_{k-1}\right), \beta_{-1}=1, \beta_{k}=\prod_{j=0}^{k} x_{j}$ and $A_{1 / 2}$ is the nearest integer Continued Fraction map. In $\S 2.1$ we will give a brief account of useful facts concerning continued fractions.

In Marmi et al. 2001 the complex Bruno function has been introduced ${ }^{2}$, more precisely authors defined an analytic map $\mathbf{B}: \mathbb{H}^{+} \rightarrow \mathbb{H}^{+}$, where $\mathbb{H}^{+}$is the upper Poincaré half plane, $\mathbb{Z}$-periodic, which verifies a functional equation similar to the one for the 1-Bruno function. The boundary behavior of $\mathbf{B}$ is given by (see Theorem 5.19 and $\$ 5.2 .9$ of Marmi et al. 2001):

(1) let $H>0$, then the imaginary part of $\mathbf{B}(z)$ tends to $B_{1 / 2}(\Re z)$ when $\Im z \rightarrow 0$ and $z \in\left\{\zeta \in \mathbb{H}^{+}: \Im \zeta \geq|\Re \zeta|^{H}\right\}$, whenever $\Re z \in \mathcal{B}$;

(2) $\Re \mathbf{B}(z)$ is bounded on $\mathbb{H}^{+}$, its trace is continuous at irrational points and it has a jump of $\pi / q$ for $\Re z=p / q \in \mathbb{Q}$.

In section 2 we introduce an explicit formula for the 1/2-complex Bruno function which corrects a small error in $\S$ A.4.4 page 836, and gives more details than Appendix A.4 of Marmi et al. 2001. We will also give an algorithm to compute it numerically.

1.1. The Yoccoz function. We already observed that the function $B_{1 / 2}$ is related to the stability problem of a fixed point of an analytic diffeomorphism of $\mathbb{C}$, in the rest of this section we will show this relation by describing the Yoccoz result (Yoccoz 1995, Chapter II). Let $\lambda \in \mathbb{C}^{*}$ and let us consider the quadratic polynomial $P_{\lambda}(z)=\lambda z(1-z)$. The origin is a fixed point and we are interested in studying its stability. If $|\lambda|<1$ (hyperbolic case) then it follows from the results of Poincaré and Koenigs that the origin is stable, whereas if $\lambda=e^{2 \pi i p / q}$ (parabolic case) the origin is not stable.

Let now consider $\lambda \in \mathbb{D}^{*}$ and let $H_{\lambda}(z)$ be the conformal map which locally linearizes $P_{\lambda}$ (its existence is guaranteed by the Poincaré-Koenigs results):

$$
P_{\lambda} \circ H_{\lambda}=H_{\lambda} \circ R_{\lambda},
$$

where $R_{\lambda}(z)=\lambda z$, and let us denote by $r_{2}(\lambda)$ the radius of convergence of $H_{\lambda}$.

One can prove that $H_{\lambda}$ can be analytically continued to a larger set, the basin of attraction of $0:\left\{z \in \mathbb{C}: P_{\lambda}^{\circ n}(z) \rightarrow 0, n \rightarrow+\infty\right\}$, but not to the whole of $\mathbb{C}$, and it has a unique singular point on its circle of convergence $\mathbb{D}_{r_{2}(\lambda)}$, which will be denoted by $U(\lambda) \in \mathbb{C}$. The function $U: \mathbb{D}^{*} \rightarrow \mathbb{C}$ is called the Yoccoz function.

Yoccoz proved that $U$ has an analytic bounded extension to $\mathbb{D}$ and moreover it can be obtained as limit of polynomials $U_{n}(\lambda)=\lambda^{-n} P_{\lambda}^{\circ n}\left(z_{\text {crit }}\right)$, uniformly over compact subsets of $\mathbb{D}$, where $z_{\text {crit }}=1 / 2$ is the critical point of the quadratic polynomial. Being this extension not identically zero, by a classical result of Fatou, the Yoccoz function has radial limits almost everywhere, and the set $\lambda_{0} \in S^{1}$ for which $\lim \sup _{\lambda \rightarrow \lambda_{0}} U(\lambda)=0$ has zero measure. This means that the quadratic

\footnotetext{
${ }^{2}$ Following the notation introduced for the real Bruno functions, we should call this complex extension the 1-complex Bruno function. In fact we will see at the end of section 2 that it is constructed "following" the Gauss continued fraction algorithm. In this way we could also distinguish it from the 1/2-complex Bruno function that we will introduce in section[2] "following" the nearest integer continued fraction algorithm.
} 
polynomial is linearizable $\left(r_{2}\left(\lambda_{0}\right)=\left|U\left(\lambda_{0}\right)\right|>0\right)$ for a full measure set of $\lambda_{0} \in S^{1}$, but the proof doesn't give any information on this set.

When $|\lambda|=1$ and $\lambda$ is not a root of the unity, assuming $\lambda=e^{2 \pi i \omega}$, for some irrational $|\omega|<1 / 2$, Yoccoz proved (Yoccoz 1995, Theorem 1.8 Chapter II) that $P_{\lambda}(z)$ is linearizable if and only if $\omega \in \mathcal{B}_{1 / 2}$, moreover there exists a constant $C_{1}$, and for all $\epsilon>0$ a constant $C(\epsilon)$ such that for all $\omega \in \mathcal{B}_{1 / 2}$ :

$$
C_{1} \leq \log r_{2}\left(e^{2 \pi i \omega}\right)+B_{1 / 2}(\omega) \leq C(\epsilon)+\epsilon B_{1 / 2}(\omega) .
$$

We are then interested in studying the function $\omega \mapsto \log \left|U\left(e^{2 \pi i \omega}\right)\right|+B_{1 / 2}(\omega)$ and some "natural" questions arise (Yoccoz 1995 §3.2 page 72):

(Yoccoz's Conjecture). Is the function $\omega \mapsto \log \left|U\left(e^{2 \pi i \omega}\right)\right|+B_{1 / 2}(\omega)$

bounded for $\omega \in \mathbb{R}$ ?

Motivated by numerical results of Marmi 1990 and by some analytic properties of the real Bruno function (see the following Remark 1.1] and [Marmi et al. 1997]) it has been conjectured that:

(Marmi-Moussa-Yoccoz's Conjecture). The function, defined on the set of Bruno number, $\omega \mapsto \log \left|U\left(e^{2 \pi i \omega}\right)\right|+B_{1 / 2}(\omega)$, extends to a $1 / 2$-Hölder continuous function on $\mathbb{R}$.

Remark 1.1 (Why 1/2-Hölder?). In Marmi et al. 1997] authors proved a "stability result" for $B_{1 / 2}$ (\$4 page 285). Let us rewrite the functional equation for the 1/2-Bruno function as follow:

$$
\left[B_{1 / 2}(x)-x B_{1 / 2}\left(x^{-1}\right)\right]=-\log x
$$

if we add to the r.h.s. a "regular term" $f$, say $\eta$-Hölder continuous, and we call $B_{f}$ the solution of:

$$
\left[B_{f}(x)-x B_{f}\left(x^{-1}\right)\right]=-\log x+f(x),
$$

then $B_{1 / 2}-B_{f}$ is $1 / 2-$ Hölder continuous if $f$ is at least $1 / 2-$ Hölder. Hence if we prove ${ }^{3}$ that the function $\omega \mapsto\left[\log \left|U\left(e^{2 \pi i \omega}\right)\right|-\omega \log \left|U\left(e^{2 \pi i \omega^{-1}}\right)\right|\right]-\log \omega$ is Hölder continuous with exponent $\eta \geq 1 / 2$, for $\omega \in[0,1 / 2]$, then the Conjecture holds.

Very recently X. Buff and A. Cheritat Buff-Cheritat 2003 proved the Yoccoz conjecture. Whereas the Marmi-Moussa-Yoccoz conjecture is still open. We will be interested in the following conjecture, equivalent to the one of Marmi-MoussaYoccoz:

(Marmi-Moussa-Yoccoz's Conjecture) The analytic function, defined on the upper Poincaré half plane, $z \mapsto \mathcal{H}(z)=\log U\left(e^{2 \pi i z}\right)-$

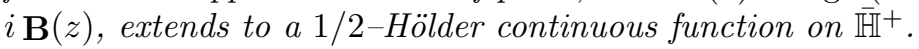

The aim of this paper is twofold. First give more insight into the $1 / 2-$ complex Bruno function and second to make a first step toward the understanding of the last conjecture. Our numerical results allows to conclude that $\mathcal{H}$ is $\eta$-Hölder continuous and we obtain an estimate of the Hölder exponent $\eta=0.498 \pm 0.004$. This gives us good numerical evidence that the Marmi-Moussa-Yoccoz conjecture should be true.

\footnotetext{
${ }^{3}$ Transform a function according to $\psi(x) \mapsto \psi(x)-x \psi(1 / x)$ to "reduce the strength of singularities" is the main idea of the Modular Smoothing. We refer to Buric et al. 1990 where authors describe the method and apply it to the critical function of the Semistandard Map.
} 
The paper is organized as follows: in section 2 we introduce the $1 / 2$-complex Bruno function and some results from number theory (approximations of rationals by rationals), to obtain an algorithm to compute the complex Bruno function. In section 3 we explain how to calculate the Yoccoz function and then, after a brief introduction of the Littlewood-Paley Theory in $\S$, used to test the Hölder continuity, we present our results in section 5 Appendix collects some considerations related to technical aspects of our numerical test.

Acknowledgments. I am grateful to Jacques Laskar for putting at my disposal a large amount of CPU times on computers of Astronomie et Systèmes Dynamiques Team at IMCCE Paris.

\section{The 1/2-Complex Bruno functions}

The aim of this section is to introduce, starting from Appendix A.4 of Marmi et al. 2001, a complex extension of the 1/2-real Bruno function and to give an algorithm to compute it numerically.

Let us consider $f \in L^{2}([0,1 / 2])$, extended: 1-periodic, $f(x+1)=f(x)$ for all $x \in \mathbb{R}$, and even $f(x)=f(-x)$ for all $x \in[-1 / 2,0]$, and then let us introduce the operator $T$ acting on such $f$ by:

$$
T f(x)=x f\left(\frac{1}{x}\right)
$$

we remark that the functional equation (1.1) can be rewritten as:

$$
(1-T) B_{1 / 2}(x)=-\log x \quad \forall x \in(0,1 / 2) .
$$

Let $\left(T_{m}\right)_{m \geq 2}$ be the operators defined by:

$$
\left(T_{m} f\right)(x)= \begin{cases}x f\left(\frac{1}{x}-m\right) & x \in\left(\frac{1}{m+1 / 2}, \frac{1}{m}\right) \\ x f\left(m-\frac{1}{x}\right) & x \in\left(\frac{1}{m}, \frac{1}{m-1 / 2}\right] \text { branch } m^{+} \\ \text {branch } m^{-} & \text {otherwise }\end{cases}
$$

then using the periodicity and the evenness of $f$, we can rewrite (2.1) as follows:

$$
T f(x)=\sum_{m \geq 2}\left\{x f\left(\frac{1}{x}-m\right)+x f\left(m+1-\frac{1}{x}\right)\right\} .
$$

To introduce the 1/2-complex Bruno function we have to extend (2.4) to complex analytic functions; this is done Marmi et al. 2001 by considering the complex vector space of holomorphic functions in $\overline{\mathbb{C}} \backslash[0,1 / 2]$, vanishing at infinity: $\mathcal{O}^{1}(\overline{\mathbb{C}} \backslash[0,1 / 2])$ (which is isomorphic to the space of hyperfunctions with support contained in $[0,1 / 2])$. So, let $\varphi$ be the Hilbert transform of $f$ :

$$
\varphi(z)=\frac{1}{\pi} \int_{0}^{1 / 2} \frac{f(x)}{x-z} d x,
$$

then starting from (2.4) we define the action of $T$ on $\varphi$ as follows:

$$
T \varphi(z)=\sum_{m \geq 2} L_{g(m)}\left(1+L_{\sigma}\right) \varphi(z),
$$


where $g(m)=\left(\begin{array}{ll}0 & 1 \\ 1 & m\end{array}\right), \sigma=\left(\begin{array}{cc}-1 & 1 \\ 0 & 1\end{array}\right)$ and $L_{\left(\begin{array}{ll}a & b \\ c & d\end{array}\right)}$ acts on $\mathcal{O}^{1}(\overline{\mathbb{C}} \backslash[0,1 / 2])$ by:

$$
L_{\left(\begin{array}{ll}
a & b \\
c & d
\end{array}\right)} \varphi(z)=(a-c z)\left[\varphi\left(\frac{d z-b}{a-c z}\right)-\varphi\left(-\frac{d}{c}\right)\right]-\frac{a d-b c}{c} \varphi^{\prime}\left(-\frac{d}{c}\right) .
$$

In the spirit of (2.2) we want to consider $(1-T)^{-1}$ acting on some $\varphi \in \mathcal{O}^{1}(\overline{\mathbb{C}} \backslash[0,1 / 2])$, and to obtain a $\mathbb{Z}$-periodic, "even function" ${ }^{4}$, we will consider:

$$
\sum_{n \in \mathbb{Z}}\left[\left(1+L_{\sigma}\right)(1-T)^{-1}\right] \varphi(z-n) .
$$

Let us introduce the operator $\hat{T}$ defined by $\left(1+L_{\sigma}\right) T=\hat{T}\left(1+L_{\sigma}\right)$, then from (2.5) and the relation: $(1-T)^{-1}=\sum_{r \geq 0} T^{r}$, we can expand $(1-\hat{T})^{-1}$ in terms of matrices $g(m)$ and $\sigma$, to obtain a sum of matrices of the form: $\epsilon_{0} g\left(m_{1}\right) \ldots \epsilon_{r-1} g\left(m_{r}\right)$, where $r \geq 1, m_{i} \geq 2$ and $\epsilon_{i-1} \in\{1, \sigma\}$, for $1 \leq i \leq r$.

Let us set $\hat{\mathcal{M}}^{(0)}=\{1\}$ and for $r \geq 1$ :

$$
\begin{array}{r}
\hat{\mathcal{M}}^{(r)}=\left\{g \in G L(2, \mathbb{Z}): \exists \epsilon_{0}, \ldots, \epsilon_{r-1}\right. \\
\in\{1, \sigma\}, m_{1}, \ldots, m_{r} \geq 2 \quad: \\
\left.g=\epsilon_{0} g\left(m_{1}\right) \ldots \epsilon_{r-1} g\left(m_{r}\right)\right\},
\end{array}
$$

and finally $\hat{\mathcal{M}}=\cup_{r \geq 0} \hat{\mathcal{M}}^{(r)}$ : the $1 / 2$-Monoid (we left to $\S 2.3$ a more detailed discussion of this monoid and the reason of its name).

It remains to specify the "good" $\varphi \in \mathcal{O}^{1}(\overline{\mathbb{C}} \backslash[0,1 / 2])$ to apply $(2.7)$, to have the wanted properties for $\mathbf{B}$. This is done by considering the Hilbert transform of the even, 1-periodic logarithm function defined in $(0,1 / 2]$, namely:

$$
\begin{aligned}
\varphi_{1 / 2}(z) & =\frac{1}{\pi} \int_{0}^{1 / 2} \frac{-\log x}{x-z} d x \\
& =-\frac{1}{\pi} L i_{2}\left(\frac{1}{2 z}\right)+\frac{1}{\pi} \log 2 \log \left(1-\frac{1}{2 z}\right),
\end{aligned}
$$

where $L i_{2}(z)$ is the dilogarithm function Oesterlé 1993: the analytic continuation of $\sum_{n \geq 1} z^{n} n^{-2}$, to $\mathbb{C} \backslash[1,+\infty)$. We are now able to define the $1 / 2$-complex Bruno function to be:

$$
\mathbf{B}(z)=\sum_{n \in \mathbb{Z}}\left[\sum_{g \in \hat{\mathcal{M}}} L_{g}\left(1+L_{\sigma}\right)\right] \varphi_{1 / 2}(z-n) .
$$

This formula defines ${ }^{5}$ an holomorphic function, defined in $\mathbb{H}^{+}, \mathbb{Z}$-periodic, with an even imaginary part for $\Re z \in[0,1 / 2]$, and then ${ }^{6}$ an odd real part, on the same domain.

Remark 2.1. The 1/2-complex Bruno function is 1-periodic and so we can consider its Fourier series: $\mathbf{B}(z)=\sum_{l \in \mathbb{Z}} \hat{b}_{l} e^{2 \pi i l z}$. Introducing the variable $w=e^{2 \pi i z}$ the Bruno function is mapped into an analytic function, $\tilde{\mathbf{B}}(w)$, defined in $\mathbb{D}^{*}$, which can be extended by continuity to $\mathbb{D}$. Its Taylor series at the origin is $\tilde{\mathbf{B}}(w)=$

\footnotetext{
${ }^{4}$ Here and in the following by even complex function we will mean even w.r.t. $\Re z \rightarrow-\Re z$.

${ }^{5}$ This claim can be obtained by slightly modification of the proof given in Marmi et al. 2001] for the 1-Complex Bruno function and we omit it referring to Marmi et al. 2001 for any details.

${ }^{6}$ This is a standard result for harmonic conjugate functions. For this reason we prefer to speak of 1/2-Complex Bruno's function instead of even Complex Bruno's function.
} 
$\sum_{l \in \mathbb{N}} \hat{b}_{l} w^{l}$, hence Fourier coefficients of $\mathbf{B}(z)$ corresponding to negative modes are all identically zero. Moreover, because of the parity properties of $\Re \mathbf{B}$ and $\Im \mathbf{B}$, its Fourier coefficients are all purely imaginary, in fact:

$$
\hat{b}_{l}=2 i \int_{0}^{1 / 2}[-\sin (2 \pi l x) \Re \mathbf{B}(x+i t)+\cos (2 \pi l x) \Im \mathbf{B}(x+i t)] d x .
$$

The goal of the next sections will be to express (2.10) in terms of a sum over a class of rational numbers in such a way we could give ( $\$ 2.4$ ) an algorithm to compute it. This will be accomplished thanks to a new characterization $(\S$ 2.3) of the $1 / 2-$ Monoid $\hat{\mathcal{M}}$, after having introduced some results from Number Theory: $\S 2.1$ Continued Fraction Theory and $\S 2.2$ Farey Series.

2.1. Continued Fraction. We consider the so called nearest integer Continued Fraction algorithm ${ }^{7}$. We state here some basic facts we will need in the following and we refer to Hardy-Wright 1979, Marmi et al. 1997] for a more complete discussion. Let $\|x\|=\min _{p \in \mathbb{Z}}\{x<1 / 2+p\}$, then to each $x \in \mathbb{R}$ we associate a continued fraction as follows:

$$
a_{0}=\|x\|, \quad x_{0}=\left|x-a_{0}\right|, \quad \varepsilon_{0}=\left\{\begin{array}{l}
+1 \text { iff } x \geq a_{0} \\
-1 \text { otherwise }
\end{array},\right.
$$

and then inductively for all $n \geq 0$, as long as $x_{n} \neq 0$ :

$a_{n+1}=\left\|x_{n}^{-1}\right\|, \quad x_{n+1}=\left|x_{n}^{-1}-a_{n+1}\right| \equiv A_{1 / 2}\left(x_{n}\right), \quad \varepsilon_{n+1}=\left\{\begin{array}{l}+1 \text { iff } x_{n}^{-1} \geq a_{n+1} \\ -1 \text { otherwise }\end{array}\right.$.

We will use the standard compact notation to denote the continued fraction $x=$ $\left[\left(a_{0}, \varepsilon_{0}\right), \ldots,\left(a_{n}+\varepsilon_{n} x_{n}, \varepsilon_{n}\right)\right]$. From the definition it follows that $x_{n}>2$ and so $a_{n} \geq 2$.

Remark 2.2 (Standard form for finite continued fraction). Let $\left[\left(a_{0}, \varepsilon_{0}\right), \ldots,\left(a_{\bar{n}}, \varepsilon_{\bar{n}}\right)\right]$ be a finite continued fraction of length $\bar{n}$. Then, whenever $a_{\bar{n}}=2$, we must also have $\varepsilon_{\bar{n}-1}=+1$, namely $\left[\left(a_{0}, \varepsilon_{0}\right), \ldots,\left(a_{\bar{n}-1},-1\right),(2,+1)\right]$ represents the same rational number that $\left[\left(a_{0}, \varepsilon_{0}\right), \ldots,\left(a_{\bar{n}-1}-1,+1\right),(2,+1)\right]$. Moreover a finite continued fraction cannot contain a couple $\left(a_{l}, \varepsilon_{l}\right)=(2,-1)$ for any $l \leq \bar{n}$.

We recall, without proof some known results:

- the continued fraction algorithm stops if and only if $x \in \mathbb{R} \backslash \mathbb{Q}$, this correspondence in bijective up to the standard convention of Remark 2.2.

- For any positive integer $n$ (or smaller than the length of the finite continued fraction) the $n^{\text {th }}$ convergent is defined by:

$$
\frac{p_{n}}{q_{n}}=\left[\left(a_{0}, \varepsilon_{0}\right), \ldots,\left(a_{n}, \varepsilon_{n}\right)\right],
$$

one can prove that $p_{n}$ and $q_{n}$ are recursively defined by:

$$
\left\{\begin{array}{l}
p_{n}=a_{n} p_{n-1}+\varepsilon_{n-1} p_{n-2} \\
q_{n}=a_{n} q_{n-1}+\varepsilon_{n-1} q_{n-2},
\end{array}\right.
$$

starting with $p_{-1}=q_{-2}=1, p_{-2}=q_{-1}=0$ and $\varepsilon_{-1}=1$.

\footnotetext{
${ }^{7}$ In Marmi et al. 1997 a one parameter family of continued fraction developments has been introduced. The nearest integer continued fraction corresponds to the value $1 / 2$ of the parameter, so we will also call it $1 / 2-$ continued fraction.
} 
- for all $n$ we have: $q_{n} p_{n-1}-p_{n} q_{n-1}=(-1)^{n} \varepsilon_{0} \ldots \varepsilon_{n-1}$.

2.2. The Farey Series. Let $n \in \mathbb{N}^{*}$, the Farey Series Hardy-Wright 1979 of order $n$ is the set of irreducible fractions in $[0,1]$ whose denominators do not exceed $n^{8}$ :

$$
\mathcal{F}_{n}=\{p / q \in[0,1]:(p, q)=1 \text { and } q \leq n\} .
$$

The cardinality of $\mathcal{F}_{n}$ is given by the Euler $\phi(n)$ function and so it is asymptotic to $3 n^{2} / \pi^{2}$ for $n$ large. The Farey Series is characterized by the following two properties Hardy-Wright 1979:

Theorem 2.3. Let $n \geq 1$. If $p / q$ and $p^{\prime} / q^{\prime}$ are two successive elements of $\mathcal{F}_{n}$, then: $q p^{\prime}-q^{\prime} p=1$.

Theorem 2.4. Let $n \geq 1$. If $p^{\prime} / q^{\prime}, p / q$ and $p^{\prime \prime} / q^{\prime \prime}$ are three successive elements (in this order) of $\mathcal{F}_{n}$, then:

$$
\frac{p}{q}=\frac{p^{\prime}+p^{\prime \prime}}{q^{\prime}+q^{\prime \prime}}
$$

Using an idea contained in the proof of Theorem 2.4 given in Hardy-Wright 1979, we construct an algorithm (easily implementable on a computer) which allows us to carry out for any $n \geq 2$ the Farey Series of order $n$. Using Proposition 2.6 we will give a second algorithm to compute the Farey series up to any given order $n$, using the continued fraction development.

Proposition 2.5 (Construction of $\mathcal{F}_{n}$ ). Let $n \geq 2$, then the elements of $\mathcal{F}_{n}$, $\left(p_{i} / q_{i}\right)_{1 \leq i \leq \phi(n)}$, are recursively defined by:

$$
\left\{\begin{array}{l}
p_{i+1}=-p_{i-1}+r_{i} p_{i} \\
q_{i+1}=-q_{i-1}+r_{i} q_{i},
\end{array}\right.
$$

where $r_{i}=\left\lfloor\left(n+q_{i-1}\right) / q_{i}\right\rfloor$, starting with $\left(p_{1}, q_{1}\right)=(0,1),\left(p_{2}, q_{2}\right)=(1, n)$ and $\left(p_{3}, q_{3}\right)=(1, n-1)$.

Proof. Let $p / q \in \mathcal{F}_{n}$. Because $p$ and $q$ are relatively prime we can always solve in $\mathbb{Z}^{2}$ the linear Diophantine equation $q P-p Q=1$ : let $\left(P_{0}, Q_{0}\right)$ be a particular solution and let $r$ be the integer such that: $n-q<Q_{0}+r q \leq n$, namely $r=\left\lfloor\left(n-Q_{0}\right) / q\right\rfloor$.

Let us define $P_{r}=P_{0}+r p$ and $Q_{r}=Q_{0}+r q$, then the following claims are trivial: $\left(P_{r}, Q_{r}\right)$ is again a solution of the linear diophantine equation, $\left(P_{r}, Q_{r}\right)=1$ and $0<Q_{r} \leq n$. So $P_{r} / Q_{r} \in \mathcal{F}_{n}$. Clearly $P_{r} / Q_{r}>p / q$ and we claim that it is the immediate successor of $p / q$ in $\mathcal{F}_{n}$.

To obtain a constructive algorithm we must solve the linear diophantine equation, this is achieved by considering the element which precedes $p / q$ in $\mathcal{F}_{n}$ : let us denote it by $p^{\prime} / q^{\prime}$. A particular solution is then given by $P_{0}=-p^{\prime}, Q_{0}=-q^{\prime}$ : from

\footnotetext{
${ }^{8}$ This is different from the Farey Tree which is still a set of rational numbers in $[0,1]$ which can be constructed by induction starting with: $\hat{\mathcal{F}}_{0}=\{0,1\}$ and then defining the $i$-th element of $\hat{\mathcal{F}}_{n}, n \geq 1$, by:

$$
\frac{\hat{p}_{i}^{(n)}}{\hat{q}_{i}^{(n)}}=\frac{\hat{p}_{i-1}^{(n-1)}+\hat{p}_{i}^{(n-1)}}{\hat{q}_{i-1}^{(n-1)}+\hat{q}_{i}^{(n-1)}} .
$$

The Farey Tree of order $n$ is clearly larger than the corresponding Farey Series and $\operatorname{card} \hat{\mathcal{F}}_{n}=$ $2^{n}+1$.
} 
the previous result the element following $p / q$ is then given by: $P_{r}=-p^{\prime}+r p$, $Q_{r}=-q^{\prime}+r q$, where $r=\left\lfloor\left(n+q^{\prime}\right) / q\right\rfloor$.

To finish the algorithm we need two starting elements of $\mathcal{F}_{n}$ apart of $0 / 1$, but it is easy to realize that the first three elements of $\mathcal{F}_{n}$ are $0 / 1,1 / n$ and $1 /(n-1)$, whenever $n \geq 2$.

We are now able to give a second algorithm to construct the Farey Series of order $n$. Here is the idea: given an irreducible fraction $p / q \in(0,1)$, we compute its continued fraction development and then following two rules: Truncate and Subtract one, we obtain two new irreducible fractions in $[0,1]$ which will result to be the predecessor and the successor of $p / q$ in $\mathcal{F}_{n}$ with $n=q$.

Proposition 2.6 (Construction of $\mathcal{F}_{n}, 2^{\text {nd }}$ version). Let $p / q \in(0,1)$ and let $p^{\prime} / q^{\prime}<$ $p / q<p^{\prime \prime} / q^{\prime \prime}$ be three successive elements of $\mathcal{F}_{q}$. Assume $p / q=\left[\left(a_{0}, \varepsilon_{0}\right), \ldots,\left(a_{\bar{n}}, \varepsilon_{\bar{n}}\right)\right]$ for some $\bar{n} \geq 1$ and let us define the rational numbers $p_{T} / q_{T}$ and $p_{S} / q_{S}$ as follows ${ }^{9}$ :

$$
\frac{p_{T}}{q_{T}}=\left[\left(a_{0}, \varepsilon_{0}\right), \ldots,\left(a_{\bar{n}-1}, \varepsilon_{\bar{n}-1}\right)\right] \quad \text { (Truncate) },
$$

and

$$
\frac{p_{S}}{q_{S}}=\left[\left(a_{0}, \varepsilon_{0}\right), \ldots,\left(a_{\bar{n}}-1, \varepsilon_{\bar{n}}\right)\right] \quad \text { (Subtract one) } .
$$

Then if $\varepsilon_{0} \ldots \varepsilon_{\bar{n}-1}=+1$, we have

$$
\begin{cases}p_{T} / q_{T}=p^{\prime} / q^{\prime}, \text { and }, p_{S} / q_{S}=p^{\prime \prime} / q^{\prime \prime} & \text { if } \bar{n} \text { is even } \\ p_{T} / q_{T}=p^{\prime \prime} / q^{\prime \prime}, \text { and, } p_{S} / q_{S}=p^{\prime} / q^{\prime} & \text { if } \bar{n} \text { is odd } .\end{cases}
$$

Whereas if $\varepsilon_{0} \ldots \varepsilon_{\bar{n}-1}=-1$ we have the symmetric case, namely

$$
\left\{\begin{array}{l}
p_{T} / q_{T}=p^{\prime \prime} / q^{\prime \prime}, \text { and, } p_{S} / q_{S}=p^{\prime} / q^{\prime} \quad \text { if } \bar{n} \text { is even } \\
p_{T} / q_{T}=p^{\prime} / q^{\prime}, \text { and, } p_{S} / q_{S}=p^{\prime \prime} / q^{\prime \prime} \quad \text { if } \bar{n} \text { is odd. }
\end{array}\right.
$$

Proof. By (2.14), (2.17) and (2.18) we have:

$$
\left\{\begin{array} { l } 
{ p _ { T } = a _ { \overline { n } - 1 } p _ { \overline { n } - 2 } + \varepsilon _ { \overline { n } - 2 } p _ { \overline { n } - 3 } } \\
{ q _ { T } = a _ { \overline { n } - 1 } q _ { \overline { n } - 2 } + \varepsilon _ { \overline { n } - 2 } q _ { \overline { n } - 3 } }
\end{array} \text { and } \left\{\begin{array}{l}
p_{S}=\left(a_{\bar{n}}-1\right) p_{\bar{n}-1}+\varepsilon_{\bar{n}-1} p_{\bar{n}-2} \\
q_{S}=\left(a_{\bar{n}}-1\right) q_{\bar{n}-1}+\varepsilon_{\bar{n}-1} q_{\bar{n}-2}
\end{array}\right.\right.
$$

then:

$$
\frac{p_{T}+p_{S}}{q_{T}+q_{S}}=\frac{a_{\bar{n}-1} p_{\bar{n}-2}+\varepsilon_{\bar{n}-2} p_{\bar{n}-3}+\left(a_{\bar{n}}-1\right) p_{\bar{n}-1}+\varepsilon_{\bar{n}-1} p_{\bar{n}-2}}{a_{\bar{n}-1} q_{\bar{n}-2}+\varepsilon_{\bar{n}-2} q_{\bar{n}-3}+\left(a_{\bar{n}}-1\right) q_{\bar{n}-1}+\varepsilon_{\bar{n}-1} q_{\bar{n}-2}}=\frac{p_{\bar{n}}}{q_{\bar{n}}}=\frac{p}{q},
$$

where we used the definition of $p / q$ with its finite continued fraction of length $\bar{n}$. Finally:

and similarly

$$
\frac{p}{q}-\frac{p_{T}}{q_{T}}=\frac{p_{\bar{n}} q_{\bar{n}-1}-p_{\bar{n}-1} q_{\bar{n}}}{q_{\bar{n}} q_{\bar{n}-1}}=\frac{(-1)^{\bar{n}+1} \varepsilon_{0} \ldots \varepsilon_{\bar{n}-1}}{q_{\bar{n}} q_{\bar{n}-1}},
$$

$$
\frac{p}{q}-\frac{p_{S}}{q_{S}}=\frac{p_{\bar{n}}\left(q_{\bar{n}}-q_{\bar{n}-1}\right)-\left(p_{\bar{n}}-p_{\bar{n}-1}\right) q_{\bar{n}}}{q_{\bar{n}} q_{S}}=\frac{(-1)^{\bar{n}} \varepsilon_{0} \ldots \varepsilon_{\bar{n}-1}}{q_{\bar{n}} q_{S}},
$$

from which the proof follows easily.

\footnotetext{
${ }^{9}$ If $a_{\bar{n}}=2$, then $\varepsilon_{\bar{n}-1}=+1$ by remark 2.2 and $p_{S} / q_{S}=\left[\left(a_{0}, \varepsilon_{0}\right), \ldots,\left(a_{\bar{n}-1}+1,+1\right)\right]$.
} 
2.3. The $1 / 2-$ Monoid. In this paragraph we will study the monoid $\hat{\mathcal{M}}$ of $G L(2, \mathbb{Z})$, introduced in (2.8) and used in the construction of the 1/2-Complex Bruno function. Our aim is to show its relation with the nearest integer continued fraction: for this reason we call it $1 / 2-$ Monoid. We will prove that given $p / q \in[0,1)$ we can "fill" the matrix $g_{*}=\left(\begin{array}{c}p_{*} \\ q_{*} \\ q\end{array}\right)$ in exactly two ways, such that it belongs to $\hat{\mathcal{M}}$ "following the nearest integer continued fraction development".

Proposition 2.7. Let $p / q \in[0,1), \bar{n} \geq 1$ and assume $p / q=\left[\left(a_{0}, \varepsilon_{0}\right), \ldots,\left(a_{\bar{n}}, \varepsilon_{\bar{n}}\right)\right]$ to be the finite continued fraction of $p / q$. We claim that the matrices $g_{T}=\left(\begin{array}{ll}p_{T} & p \\ q_{T} & q\end{array}\right)$ and $g_{S}=\left(\begin{array}{cc}p_{S} & p \\ q_{S} & q\end{array}\right)$, where the rational $p_{T} / q_{T}$ and $p_{S} / q_{S}$ have been defined in Proposition [2.6, are given by:

$$
\begin{array}{ll}
g_{T} & =\hat{\varepsilon}_{0} g\left(\hat{a}_{1}\right) \ldots g\left(\hat{a}_{\bar{n}-1}\right) \hat{\varepsilon}_{\bar{n}-1} g\left(a_{\bar{n}}\right) \\
g_{S} & =\hat{\varepsilon}_{0} g\left(\hat{a}_{1}\right) \ldots g\left(\hat{a}_{\bar{n}-1}\right) \hat{\varepsilon}_{\bar{n}-1} g\left(a_{\bar{n}}-1\right) g(1)
\end{array}
$$

where for $i=0, \ldots, \bar{n}-1$, matrices $\hat{\varepsilon}_{i}$ and integer $\hat{a}_{i}$ are defined by:

$$
\left(\hat{a}_{i}, \hat{\varepsilon}_{i}\right)=\left\{\begin{array}{ll}
\left(a_{i}, 1\right) & \text { if } \varepsilon_{i}=+1 \\
\left(a_{i}-1, \sigma\right) & \text { if } \varepsilon_{i}=-1
\end{array} .\right.
$$

Before to prove the Proposition we make the following:

Remark 2.8. It results $\hat{a}_{i} \geq 2$ for all $i$, in fact whenever $\varepsilon_{i}=-1$ one has $a_{i} \geq 3$ (see Remark 2.2). Because $p / q \in[0,1)$ the first couple $\left(a_{0}, \varepsilon_{0}\right)$ can only be one of the following two: $(0,+1)$ if $p / q \in[0,1 / 2]$ or $(1,-1)$ if $p / q \in(1 / 2,1)$.

Proof. Let $k \leq \bar{n}$ and let us introduce matrices $\hat{\varepsilon}_{0}, \ldots, \hat{\varepsilon}_{k-1}$ and integers $\hat{a}_{0}, \ldots, \hat{a}_{k}$ as in (2.23) according to the continued fraction of $p / q$. Then we claim that $g=$ $\hat{\varepsilon}_{0} g\left(\hat{a}_{1}\right) \ldots g\left(\hat{a}_{k-1}\right) \hat{\varepsilon}_{k-1} g\left(a_{k}\right)$ is equal to $\left(\begin{array}{cc}p_{k-1} & p_{k} \\ q_{k-1} & q_{k}\end{array}\right)$ where $p_{k} / q_{k}=\left[\left(a_{0}, \varepsilon_{0}\right), \ldots,\left(a_{k}, \varepsilon_{k}\right)\right]$. This can be proved by induction (use Remark 2.8 to prove the basis of induction) and then (2.21) follows by putting $k=\bar{n}$. To prove (2.22) it is enough to calculate:

$\hat{\varepsilon}_{0} g\left(\hat{a}_{1}\right) \ldots g\left(\hat{a}_{k-1}\right) \hat{\varepsilon}_{k-1} g\left(a_{k}-1\right) g(1)=\left(\begin{array}{cc}p_{k-1} & p_{k}-p_{k-1} \\ q_{k-1} & q_{k}-q_{k-1}\end{array}\right)\left(\begin{array}{cc}0 & 1 \\ 1 & 1\end{array}\right)=\left(\begin{array}{cc}p_{k}-p_{k-1} & p_{k} \\ q_{k}-q_{k-1} & q_{k}\end{array}\right)$.

Remark 2.9. Clearly matrices of type $T$ belong to $\hat{\mathcal{M}}$ (because $a_{\bar{n}} \geq 2$ ), whereas those of type $S$ belong to the monoid if and only if the continued fraction of the rational $p / q$ ends with a couple $\left(a_{\bar{n}}, \varepsilon_{\bar{n}}\right)=(2,1)$, in fact in this way the matrix $g_{S}$ is given by $\hat{\varepsilon}_{0} g\left(\hat{a}_{1}\right) \ldots g\left(\hat{a}_{\bar{n}-1}\right) g(1) g(1)=\hat{\varepsilon}_{0} g\left(\hat{a}_{1}\right) \ldots g\left(\hat{a}_{\bar{n}-1}\right) \sigma g(2)$, where we used that $\varepsilon_{\bar{n}-1}=1$ (because $a_{\bar{n}}=2$ ) and $\sigma g(m)=g(m-1) g(1)$ for all $m \geq 2$.

Remark also that, if $g$ is of type $T$ then it cannot ends with $\sigma g(2)$, in fact this will implies a continued fraction ending with $\left[\ldots,\left(a_{\bar{n}-1},-1\right),(2,1)\right]$, but we know that this is impossible and so: or $\varepsilon_{\bar{n}-1}=-1, a_{\bar{n}} \geq 3$ and $g_{T}=\ldots \sigma g\left(a_{\bar{n}}\right)$, either $\varepsilon_{\bar{n}-1}=+1, a_{\bar{n}} \geq 2$ and $g_{T}=\ldots g\left(a_{\bar{n}-1}\right) g\left(a_{\bar{n}}\right)$.

With the following proposition we will prove that $\hat{\mathcal{M}}$ is the union of matrices of type $\mathrm{T}$ and of type $\mathrm{S}$ with $\left(a_{\bar{n}}, \varepsilon_{\bar{n}}\right)=(2,1)$. Let us denote by $\mathcal{M}_{T}$ the monoid of matrices of type $\mathrm{T}$ and $\mathcal{M}_{S}$ those of type $\mathrm{S}$, with $\left(a_{\bar{n}}, \varepsilon_{\bar{n}}\right)=(2,1)$.

Proposition 2.10 (The $1 / 2-$ Monoid). $\hat{\mathcal{M}}=\mathcal{M}_{T} \cup \mathcal{M}_{S}$. 
Proof. Clearly $\mathcal{M}_{T} \cup \mathcal{M}_{S} \subset \hat{\mathcal{M}}$. Let us prove the other inclusion. Let $r \geq 1$, $m_{1}, \ldots, m_{r} \geq 2, \hat{\varepsilon}_{0}, \ldots, \hat{\varepsilon}_{r-1} \in\{1, \sigma\}$, such that $g=\hat{\varepsilon}_{0} g\left(m_{1}\right) \ldots \hat{\varepsilon}_{r-1} g\left(m_{r}\right) \in \hat{\mathcal{M}}$.

Let us consider two cases, first one: $\hat{\varepsilon}_{r-1}=\sigma$ and $m_{r} \geq 3$ or $\hat{\varepsilon}_{r-1}=1$ and $m_{r} \geq 2$; second case: $\hat{\varepsilon}_{r-1}=\sigma$ and $m_{r}=2$. In the former case to $g$ we associate a continued fraction by introducing, for $i=1, \ldots, r-1$ :

$\left(a_{0}, \varepsilon_{0}\right)=\left\{\begin{array}{ll}(0,+1) & \text { if } \hat{\varepsilon}_{i}=1 \\ (1,-1) & \text { if } \hat{\varepsilon}_{i}=\sigma\end{array}, \quad\left(a_{i}, \varepsilon_{i}\right)=\left\{\begin{array}{ll}\left(m_{i},+1\right) & \text { if } \hat{\varepsilon}_{i}=1 \\ \left(m_{i}+1,-1\right) & \text { if } \hat{\varepsilon}_{i}=\sigma\end{array}, \quad a_{r}=m_{r}\right.\right.$.

$\left[\left(a_{0}, \varepsilon_{0}\right), \ldots,\left(a_{r}, \varepsilon_{r}\right)\right]$ represents some rational $p / q$, let us define as before $p_{T} / q_{T}$ and then $g_{T}=\left(\begin{array}{ll}p_{T} & p \\ q_{T} & q\end{array}\right)=\hat{\varepsilon}_{0} g\left(\hat{a}_{1}\right) \ldots \hat{\varepsilon}_{r-1} g\left(\hat{a}_{r}\right)$ (by Proposition 2.7 where we also defined $\hat{a}_{i}$ 's). Observe that $\hat{a}_{i}=m_{i}$ to conclude $g=g_{T} \in \mathcal{M}_{T}$.

The second case can be treated similarly. Now to $g$ we associate the continued fraction $\left[\left(a_{0}, \varepsilon_{0}\right), \ldots,\left(a_{r-1}, 1\right),(2,1)\right]$ where, for $i=1, \ldots, r-2$ :

$\left(a_{0}, \varepsilon_{0}\right)=\left\{\begin{array}{ll}(0,+1) & \text { if } \hat{\varepsilon}_{i}=1 \\ (1,-1) & \text { if } \hat{\varepsilon}_{i}=\sigma\end{array}, \quad\left(a_{i}, \varepsilon_{i}\right)=\left\{\begin{array}{ll}\left(m_{i},+1\right) & \text { if } \hat{\varepsilon}_{i}=1 \\ \left(m_{i}+1,-1\right) & \text { if } \hat{\varepsilon}_{i}=\sigma\end{array}, a_{r-1}=m_{r-1}\right.\right.$.

Let $\left[\left(a_{0}, \varepsilon_{0}\right), \ldots,\left(a_{r-1}, 1\right),(2,1)\right]$ be some rational $p / q$, define as before $p_{S} / q_{S}=$ $\left[\left(a_{0}, \varepsilon_{0}\right), \ldots,\left(a_{r-1}, 1\right),(1,1)\right]$, then by Proposition 2.7

$$
g_{S}=\hat{\varepsilon}_{0} g\left(\hat{a}_{1}\right) \ldots g\left(\hat{a}_{r-1}\right) g(1) g(1)=\hat{\varepsilon}_{0} g\left(\hat{a}_{1}\right) \ldots g\left(\hat{a}_{r-1}\right) \sigma g(2)=g
$$

and it belongs to $\mathcal{M}_{S}$.

To end this section we introduce a third characterization of the $1 / 2$-Monoid, which corrects a small error in $\S$ A.4.4 page 836 of Marmi et al. 2001, and which will be useful to construct the numerical algorithm for the $1 / 2-$ complex Bruno function.

Proposition 2.11. Let $g=\left(\begin{array}{ll}a & b \\ c & d\end{array}\right) \in G$. Then $g$ belongs to $\hat{\mathcal{M}}$ if and only if $d \geq b>0, c \geq a \geq 0$ and $d \geq \mathcal{G} c$, where $\mathcal{G}=(\sqrt{5}+1) / 2$.

The proof can be done by direct computation and we omit it. We end this part with the following:

Remark 2.12 (The Gauss Monoid). In Marmi et al. 2001 authors considered the complex Bruno function constructed using the Monoid $\mathcal{M}$ :

$$
\mathcal{M}=\left\{g=\left(\begin{array}{ll}
a & b \\
c & d
\end{array}\right) \in G: d \geq b \geq a \geq 0 \text { and } d \geq c \geq a\right\} .
$$

We recall that according to the Gauss continued fraction algorithm we always have $\varepsilon_{l}=+1$, we can then prove modified version of Propositions [2.6] and 2.7 to conclude that $\mathcal{M}$ is constructed "following" the Gauss continued fraction algorithm: starting from $p / q \in(0,1)$, we complete the matrix $g_{*}=\left(\begin{array}{c}p_{*} \\ q_{*} \\ q\end{array}\right)$ into $g_{S}$ and $g_{T}$, where $p_{S} / q_{S}$ and $p_{T} / q_{T}$ are obtained with the Truncate and Subtract operations acting on the Gauss finite continued fraction of $p / q$.

2.4. An Algorithm for the 1/2-complex Bruno function. Using the results of the previous sections we are now able to give an algorithm to compute the $1 / 2-$ Complex Bruno function. Let us rewrite definition (2.10):

$$
\mathbf{B}(z)=\sum_{n \in \mathbb{Z}}\left[\sum_{g \in \hat{\mathcal{M}}} L_{g}\left(1+L_{\sigma}\right)\right] \varphi_{1 / 2}(z-n),
$$


where $\varphi_{1 / 2}(z)=-\frac{1}{\pi} L i_{2}\left(\frac{1}{2 z}\right)+\frac{1}{\pi} \log 2 \log \left(1-\frac{1}{2 z}\right)$ and the action $L_{g}$ has been defined in (2.6). From the previous sections we know that the sum over $\hat{\mathcal{M}}$ can be replaced by a sum over $p / q \in[0,1),(p, q)=1$, in such a way that to each $p / q$ we associate the matrix $g_{T}$, and also $g_{S}$ whenever the continued fraction of $p / q$ ends with $\left(a_{\bar{n}}, \varepsilon_{\bar{n}}\right)=(2,+1)$.

Using the periodicity and the parity properties of $\mathbf{B}$ we can restrict to $\Re z \in$ $[0,1 / 2]$. Let us consider the contribution of some $p / q \in[0,1)$ to $\mathbf{B}$. Because of the form of $\varphi_{1 / 2}$ and of the action $L_{g}$ we remark that the larger is the denominator of the fraction, the smaller is its contribution to the sum; moreover different rational numbers with the same denominator give comparable contributions, so we decide to order the rationals w.r.t. increasing denominators: in other words according to the Farey Series. A similar statement holds w.r.t. the sum over $\mathbb{Z}$ : large $n$ 's give small contribution to the sum. We then introduce two cut-off to effectively compute (2.10): $N_{\max }$ denoting the largest order of the Farey Series considered and $k_{1}$ the largest (in modulus) $n \in \mathbb{Z}$ which contributes to the sum over integers ${ }^{10}$.
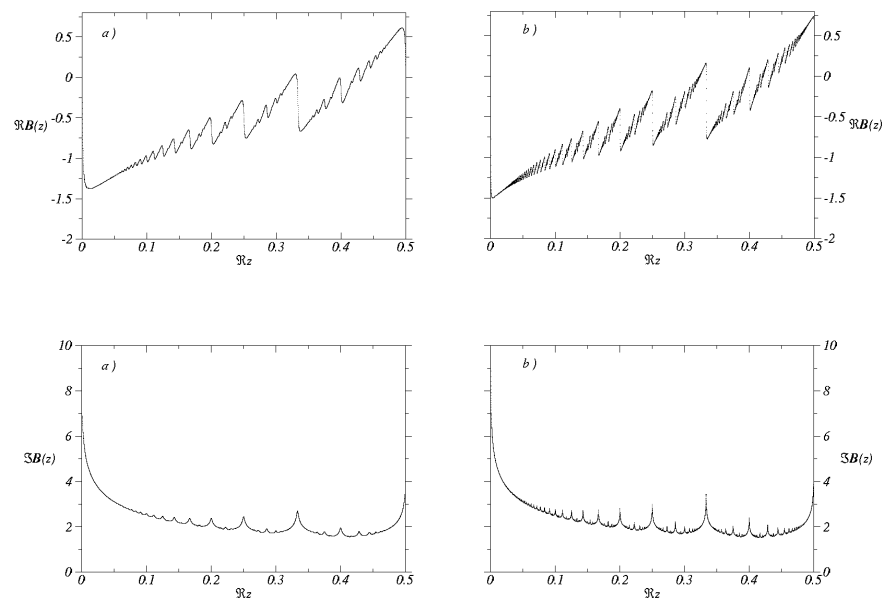

Figure 1. Plot of $\mathbf{B}(z)$ vs $\Re z$ at $\Im z$ fixed. The top line contains $\Re \mathbf{B}$ whereas on the bottom line we plot $\Im \mathbf{B}$. The column a) is for $\Im z=10^{-3}$ whereas column b) is for $\Im z=10^{-4}$. Each plot has 10000 points $\Re z$ uniformly distributed in $[0,1 / 2] . \quad k_{1}=80$, $k_{2}=20, N_{\max }=151$.

Then the 1/2-complex Bruno function can be numerically approximated by:

$$
\mathbf{B}(z) \sim \sum_{|n| \leq k_{1}} \sum_{p / q \in \mathcal{F}_{N_{\max }}}^{\prime} L_{\left(\begin{array}{c}
p_{*} \\
q_{*}
\end{array}\right)}\left(1+L_{\sigma}\right) \varphi_{1 / 2}(z-n),
$$

where $p_{*} / q_{*} \in\left\{p_{T} / q_{T}, p_{S} / q_{S}\right\}$ and the sum is restricted to fractions such that $q \geq \mathcal{G} q_{S}$, (being always $q \geq \mathcal{G} q_{T}$ ). This approximation can be made as precise as

\footnotetext{
${ }^{10}$ For technical reasons, we prefer to introduce a third cut-off, $k_{2}$. We refer to Appendix $\mathrm{A}$ to explain the role of this cut-off.
} 
we want, by choosing $N_{\max }$ and $k_{1}$ large enough, in fact (2.10) can be obtained as double limit $N_{\max } \rightarrow+\infty$ and $k_{1} \rightarrow+\infty$. In Appendix $\mathrm{A}$ we will give numerical results showing the convergence of (2.24) varying the cut-off values, the convergence of $\Im \mathbf{B}(z)$ to $B(\Re z)$ when $\Im z \rightarrow 0$ and $\Re z \in \mathcal{B}$, and the $\pi / q$-jumps of $\Re \mathbf{B}(z)$ when $z \rightarrow p / q$, as proved in Marmi et al. 2001. In Figure 1 we show some plots of $\mathbf{B}(z)$ for fixed (small) values of $\Im z$ and $\Re z \in[0,1 / 2]$, whereas in Figure 2 we show two polar plots of $e^{i \mathbf{B}(z)}$.
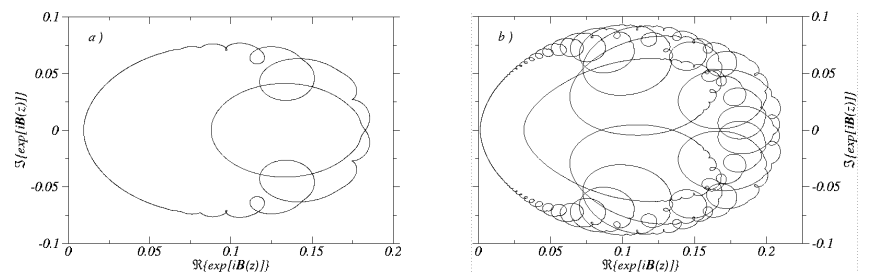

Figure 2. Polar plot of $e^{i \mathbf{B}(z)}$ for fixed values of $\Im z$. a) $\Im z=10^{-2}$ and b) $\Im z=10^{-3}$. 12000 points uniformly distributed in $[0,1]$, $k_{1}=80, k_{2}=20, N_{\max }=151$.

\section{The Yoccoz Function}

The aim of this section is to briefly introduce the algorithm used to compute the Yoccoz Function, $U(\lambda)$, introduced in $\S 1.1$ Let $\lambda \in \mathbb{D}^{*}$, let $P_{\lambda}(z)=\lambda z(1-z)$ be the quadratic polynomial and let us introduce the polynomials: $U_{n}(\lambda)=\lambda^{-n} P_{\lambda}^{\circ n}(1 / 2)$. Then we recall that the Yoccoz function is the uniform limit, over compact subsets of $\mathbb{D}$, of $U_{n}(\lambda)$.

From (1.3) and its original definition, $H_{\lambda}(U(\lambda))=1 / 2$, we get:

$$
U(\lambda)=\lambda^{-n} H_{\lambda}^{-1}\left(\lambda^{n} U_{n}(\lambda)\right),
$$

for all integer $n$. Hence to compute $U(\lambda)$ we need to know how close is $H_{\lambda}^{-1}$ to the identity, near zero and this can be done using some standard distortion estimates Buff et al. 2001. So for any fixed $\lambda \in \mathbb{D}^{*}$, we can find $n=n(\lambda)$ s.t. $P_{\lambda}^{\circ n}(1 / 2)$ is contained in some fixed disk on which we can apply the distortion estimate and then from (3.1) compute an approximation to $U(\lambda)$ with a prescribed precision $\epsilon_{U}$.

Remark 3.1 (Parity of Yoccoz's function). Let us observe the following facts. Assume $\lambda=e^{2 \pi i(x+i t)}$, with $t>0$ fixed, and $x$ varying in $(0,1 / 2)$ and let us introduce $u(x)=U\left(e^{2 \pi i(x+i t)}\right)$, to stress the dependence on $x$ only. Then we claim that $\Re u(-x)=\Re u(x)$ and $\Im u(-x)=-\Im u(x)$. The proof can be done as follows. First remark that $\lambda$, as a function of $x$, is mapped into $\bar{\lambda}$, when $x \mapsto-x$; then is enough to observe that polynomials $U_{n}(\lambda)$ verify, for $n \geq 2, U_{n}(\bar{\lambda})=\overline{U_{n}(\lambda)}$, namely:

$\Re U_{n}\left(e^{2 \pi i(x+i t)}\right)=\Re U_{n}\left(e^{2 \pi i(-x+i t)}\right)$ and $\Im U_{n}\left(e^{2 \pi i(x+i t)}\right)=-\Im U_{n}\left(e^{2 \pi i(-x+i t)}\right)$. A similar statement holds for $\log U(\lambda)$. 
Using the $\mathbb{Z}$-periodicity, we consider the Fourier series of $U\left(e^{2 \pi i z}\right)$ and using an argument similar to the one of Remark 2.1] we conclude that all the Fourier coefficients are real and zero for negative Fourier modes. Clearly Taylor's coefficients of $U(\lambda)$ coincide with Fourier coefficients of $U\left(e^{2 \pi i z}\right)$.

Figure 3 shows some polar plots of $U\left(e^{2 \pi i z}\right)$, for different values of $\Im z>0$, whereas in Figure 4 real and imaginary parts of $-\log U\left(e^{2 \pi i z}\right)$ are given. Compare with Figures 2 and 1
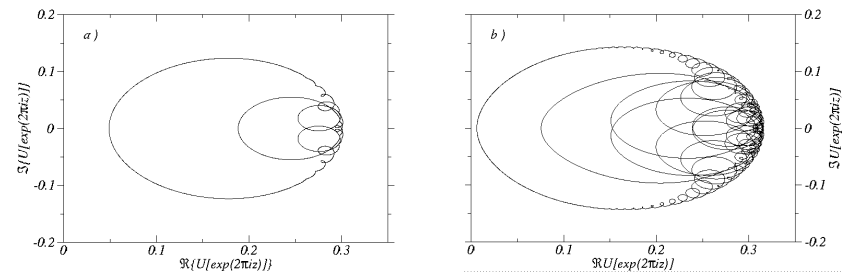

Figure 3. Polar plot of $U\left(e^{2 \pi i z}\right)$ for fixed values of $\Im z$. a) $\Im z=$ $10^{-2}$ and b) $\Im z=10^{-3}$. 12000 points uniformly distributed in $[0,1], \epsilon_{U}=10^{-3}$.
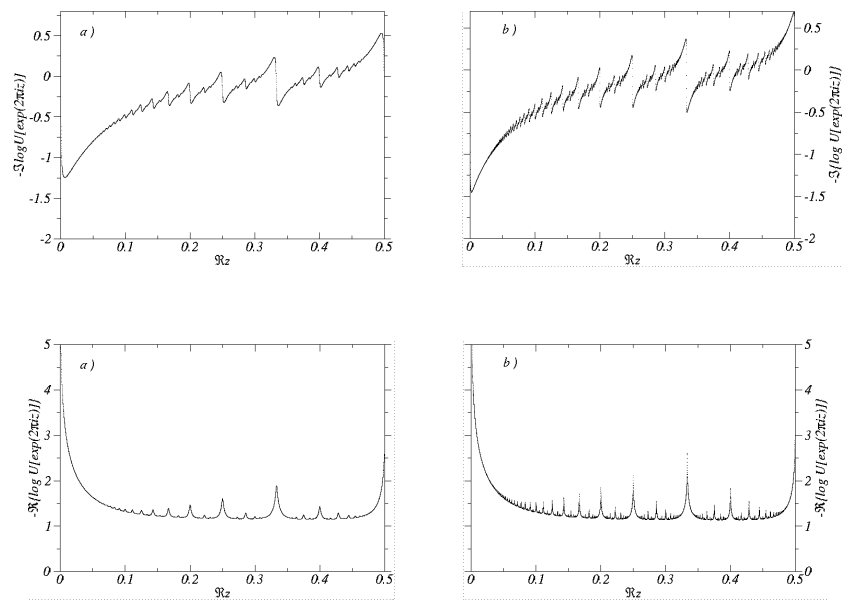

FiguRE 4. Plot of $-\log U\left(e^{2 \pi i z}\right)$ vs $\Re z$ at fixed $\Im z$. On the top we plot the imaginary part whereas on the bottom the real part. Column a) is for $\Im z=10^{-3}$, whereas on the column b) we show $\Im z=10^{-4}$ Each plot has 10000 points uniformly distributed in $[0,1 / 2], \epsilon_{U}=10^{-3}$.

Let us conclude this section with the following remark. 
Remark 3.2. In Figure 5 we show some polar plots of the "Yoccoz function" used in Buff et al. 2001] (Figure 2, page 484): they don't look like our previous pictures. Here is the reason. They take the following quadratic polynomial $Q_{\lambda}(z)=\lambda z+z^{2}$, which can be conjugate to our choice, $P_{\lambda}(z)=\lambda z(1-z)$, using $\Lambda(z)=-\lambda z$ :

$$
\Lambda \circ P_{\lambda}=Q_{\lambda} \circ \Lambda \text {. }
$$

Let us denote by $V(\lambda)$ the Yoccoz function for the polynomial $Q_{\lambda}$, then we claim that:

$$
-\lambda U(\lambda)=V(\lambda),
$$

which explain completely the relation between Figure 3 and Figure 5 . Because $-\Im \log U(\lambda)$ exhibits the same jumps at rationals as the real part of the complex Bruno function does, we choose the quadratic polynomial in the form $P_{\lambda}$.
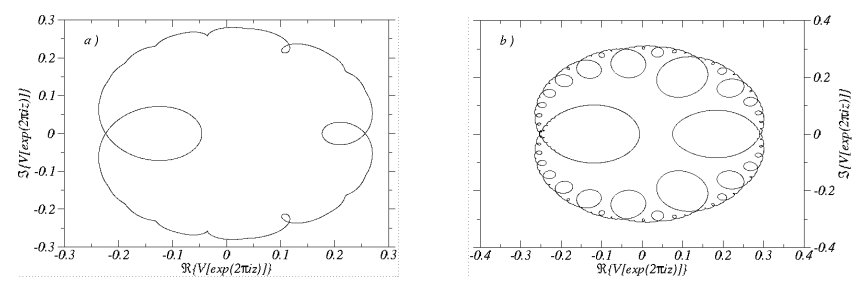

Figure 5. Polar plot of $V\left(e^{2 \pi i z}\right)$ for fixed values of $\Im z$. a) $\Im z=$ $10^{-2}$ and b) $\Im z=10^{-3}$. 12000 points uniformly distributed in $[0,1], \epsilon_{V}=10^{-3}$.

\section{The Littlewood-Paley Theory}

The aim of this section is to introduce the basic ideas and results of the LittlewoodPaley Theory, for a more complete discussion we refer to Stein 1970, Frazier et al. 1991] and also to De la Llave-Petrov 2002 where authors apply this Theory to study the regularity properties of the conjugating function for critical circle maps. In $\S 5.1$ we will present the numerical implementation of this Theory to study the Hölder regularity of the function $\mathcal{H}$ and the obtained estimate for the Hölder exponent.

The decay rate of the coefficients of a Trigonometric series, $\sum_{\mathbb{Z}} c_{k} e^{2 \pi i k x}$, does not determine whether this series is the Fourier series of some $L^{p}$ function if $p \neq 2$. More precisely given $f \in L^{p}, 1 \leq p<2$ and its Fourier series $\sum_{\mathbb{Z}} \hat{f}_{k} e^{2 \pi i k x}$, then for "almost every choice of signs \pm 1 ", the series $\sum_{\mathbb{Z}}( \pm 1) \hat{f}_{k} e^{2 \pi i k x}$ is not the Fourier series of a $L^{p}$ function. This problem has been overcome by Littlewood and Paley by "grouping together" trigonometric coefficients in dyadic blocks. Let $A>1$, $\left(\mathcal{L}_{0} f\right)(x)=\hat{f}_{0}$ and, for $M \geq 1$, let $\left(\mathcal{L}_{M} f\right)(x)=\sum_{A^{M-1} \leq|n|<A^{M}} \hat{f}_{n} e^{2 \pi i n x}$, be the dyadic partial sum of $f$. Introducing the Littlewood-Paley $d$-function: $d(f)(x)=$ $\left[\sum_{M \geq 0}\left|\mathcal{L}_{M} f(x)\right|^{2}\right]^{1 / 2}$, one can prove Littlewood-Paley 1931, Frazier et al. 1991] that for all $1<p<+\infty$ there exist positive constants $A_{p}$ and $B_{p}$ such that:

$$
A_{p}\|f\|_{p} \leq\|d(f)\|_{p} \leq B_{p}\|f\|_{p} .
$$


The Littlewood-Paley Theory is indeed more general, allowing to characterize other functional spaces by property of Fourier coefficients, for instance it applies Frazier et al. 1991] to Sobolev spaces, Hardy spaces, Hölder spaces and Besov spaces. In the case of Hölder regularity one can easily realize that Fourier coefficients of an $\eta$-Hölder continuous function decay according to $\hat{f}_{l}=\mathcal{O}\left(|l|^{-\eta}\right)$, the converse is not true but again the Littlewood-Paley Theory can characterize the Hölder regularity by the decay rate of the dyadic blocks.

An important tool in the Theory of Fourier series is the Poisson Kernel: $P_{s}(x)=$ $\sum_{k \in \mathbb{Z}} s^{|k|} e^{2 \pi i k x}, s \in[0,1)$ and $x \in \mathbb{T}$. Let $(f * g)(x)=\int_{0}^{1} f(\xi) g(x-\xi) d \xi$ be the convolution product for 1 -periodic functions. Then one can prove the following result (Stein 1970 Lemma 5 or Krantz 1983. Theorem 15.6)

Theorem 4.1 (Continuous Littlewood-Paley). Let $0<\eta<r, r \in \mathbb{N}$ and $f$ be a continuous 1-periodic function. Then $f$ is $\eta$-Hölder continuous if and only if there exists $C>0$ such that for all $t>0$ :

$$
\left\|\left(\frac{\partial}{\partial t}\right)^{r} \mathcal{P}_{f}(x, t)\right\|_{\infty} \leq C t^{\eta-r}
$$

where $\mathcal{P}_{f}(x, t)=\left(P_{\exp (-2 \pi t)} * f\right)(x)$.

We remark that if the Theorem holds for some $r \in N$, then the same is true for any $r_{1} \in \mathbb{N}, r_{1}>r$. We call this Theorem Continuous Littlewood-Paley to distinguish it from the following result, which is more close to the original idea of dyadic decomposition and we will call it Discrete Littlewood-Paley (see Krantz 1983. Theorem 5.9)

Theorem 4.2 (Discrete Littlewood-Paley). Let $\eta>0$ and let $f \in \mathcal{C}^{0}(\mathbb{T})$. Then $f$ is $\eta$-Hölder continuous function if and only if for all $A>1$ there exists a positive constant $C$ such that for all $M \in \mathbb{N}$ we have:

$$
\left\|\mathcal{L}_{M} f\right\|_{\infty} \leq C A^{-\eta M}
$$

One usually take $A=2$, and so the name dyadic decomposition, but the result is independent of the value of $A$. In the numerical implementation of this method we will use a value $A$ close to 1.25 for computational reasons.

\section{Presentation of numerical Results}

This section collects our numerical results about the Marmi-Moussa-Yoccoz conjecture that we recall here:

The analytic function, defined on $\mathbb{H}^{+}: z \mapsto \mathcal{H}(z)=\log U\left(e^{2 \pi i z}\right)-$

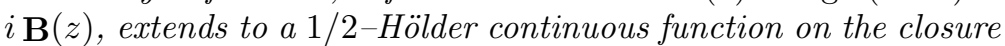
of $\mathbb{H}^{+}$.

Let us begin with some consideration concerning $\mathcal{H}$. Remark 2.1] and Remark 3.1 imply that $\mathcal{H}(z)$ has even real part for $\Re z \in[0,1 / 2]$ and an odd imaginary part on the same domain. Moreover $\mathcal{H}(z)$ is 1 -periodic and its Fourier series has only real coefficients, which correspond to non-negative Fourier modes: $\mathcal{H}(z)=\sum_{l \geq 0} \hat{h}_{l} e^{2 \pi i l z}$.

In Figure [6] we plot real and imaginary parts of $\mathcal{H}(z)$ for some fixed small $\Im z$; remark that $\mathcal{H}$ still has a "structure" but jumps of $\mathbf{B}$ and $-\log U$ seem to "compensate" to give a continuous function. The same fact holds for the "bubbles" of 

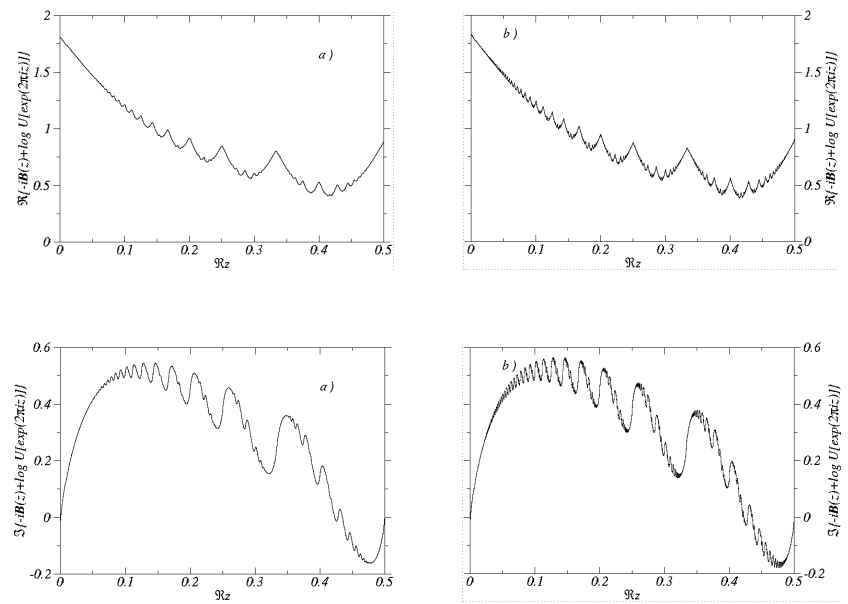

Figure 6. Plot of $-i \mathbf{B}(z)+\log U\left(e^{2 \pi i z}\right)$ vs $\Re z$ at fixed $\Im z$. On the top we show the real part whereas on the bottom the imaginary one. The first column a) is for $\Im z=10^{-3}$ and the second one b) for $\Im z=10^{-4}$. Each plot has 10000 points uniformly distributed in $[0,1 / 2] . k_{1}=80, k_{2}=20, N_{\max }=151$ and $\epsilon_{U}=10^{-3}$.

$U\left(e^{2 \pi i z}\right)$ and $e^{i \mathbf{B}(z)}$, Figure 7 show some polar plots of $e^{\mathcal{H}(z)}$ for fixed small $\Im z>0$, there are still some "bubbles" but they are far from $(0,0)$.

The Hölder continuity will be proved in the next paragraph, by giving an estimate of the Hölder exponent applying the Littlewood-Paley Theory in the Discrete and Continuous versions.
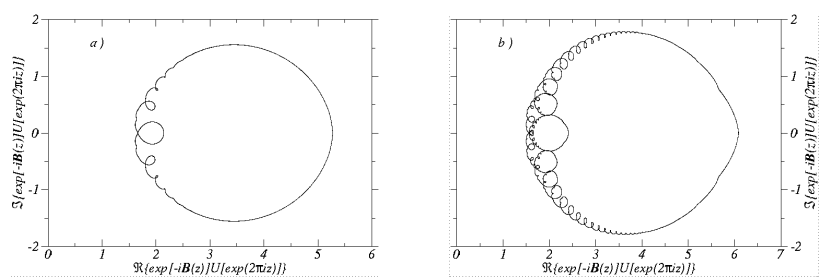

Figure 7. Polar plot of $e^{-i \mathbf{B}(z)} U\left(e^{2 \pi i z}\right)$ for fixed values of $\Im z$. a) $\Im z=10^{-2}$ and b) $\Im z=10^{-3}$. 12000 points uniformly distributed in $[0,1], k_{1}=80, k_{2}=20, N_{m}=101, \epsilon_{U}=10^{-3}$.

5.1. Numerical Littlewood-Paley method. To numerically implement the LittlewoodPaley Theory we compute from the numerical values of $\mathcal{H}$, a finite but large, number of Fourier coefficients. Then to apply Theorem 4.1 we remark that the convolution 
with the Poisson Kernel and the $r$-th derivative have the form:

$$
\left(\frac{\partial}{\partial t}\right)^{r}\left(P_{\exp (-2 \pi t)} * \mathcal{H}\right)(x)=\sum_{l \geq 0}(-2 \pi l)^{r} e^{-2 \pi t l} \hat{h}_{l} e^{2 \pi i l x}
$$

where we used the previous remark on the Fourier coefficients of $\mathcal{H}$.

We numerically compute $\left\|\left(\frac{\partial}{\partial t}\right)^{r}\left(P_{\exp (-2 \pi t)} * \mathcal{H}\right)\right\|_{\infty}$ for several small values of $t$ and some $r>1$, then applying a linear regression over the data:

$$
\log \left\|\left(\frac{\partial}{\partial t}\right)^{r}\left(P_{\exp (-2 \pi t)} * \mathcal{H}\right)\right\|_{\infty}=C_{r}^{\prime}-\beta_{C L P}(r) \log t
$$

we obtain a numerical value for $\eta_{C L P}^{(r)}=r-\beta_{C L P}(r)$.

From a numerical point of view the continuous version of the Littlewood-Paley method is better than the discrete one, in fact the former has two parameters to vary $t$ and $r$. We can vary $r$ to control if the computed value of $\eta_{C L P}^{(r)}$ stays constant or not. Moreover we can compute the l.h.s. of (5.1) for many values of $t$ and for each one all the known Fourier coefficients are used, whereas in the dyadic decomposition to "small" $M$ only "few" Fourier coefficients give their contribution and only for "large" $M$ a large number of Fourier coefficients enter.

In Figure 8 we report data from (5.1) and the corresponding linear regression values ${ }^{11}$. The estimated values of $\eta$ obtained for different $r$ are: $\eta_{C L P}^{(r=2)}=0.497 \pm$ $0.003, \eta_{C L P}^{(r=3)}=0.498 \pm 0.004$ and $\eta_{C L P}^{(r=4)}=0.498 \pm 0.003$ (errors are standard deviation errors of linear regression). They agree in the numerical precision and this gives a good indication of the validity of the results. There is no reason to prefer one value to the other and so we estimate $\eta_{C L P}=0.498 \pm 0.004$ : the mean value of the interval obtained by the union of the three intervals obtained for $r=2,3,4$.

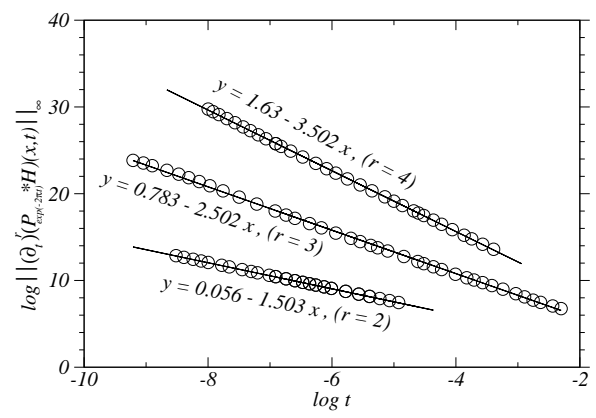

FiguRE 8. The function $\log t \mapsto \log \left\|\left(\frac{\partial}{\partial t}\right)^{r} \mathcal{P}_{\mathcal{H}}(x, t)\right\|_{\infty}$, for $r=2$, $r=3$ and $r=4$. We also show the linear regressions (5.1).

\footnotetext{
${ }^{11}$ In the Figure we decided to show only few points to have an "intelligible picture", but the linear regression are made using hundred of points.
} 
We also report the numerical results obtained using the discrete LittlewoodPaley Theorem. We fix some $A>1^{12}$ and from the computed Fourier coefficients of $\mathcal{H}$ we construct the dyadic partial sums for some large $M \in \mathbb{N}$. Then we use a linear regression on the data:

$$
\log _{A}\left\|\mathcal{L}_{M} f\right\|_{\infty}=C_{D L P}-\eta_{D L P} M,
$$

to obtain the estimate value of the Hölder coefficient: $\eta_{D L P}=0.50 \pm 0.03$ and $C_{D L P}=-4.90 \pm 0.66$. In Figure 9 we report data from (5.2) and the linear regression applied on "large $M$ ".

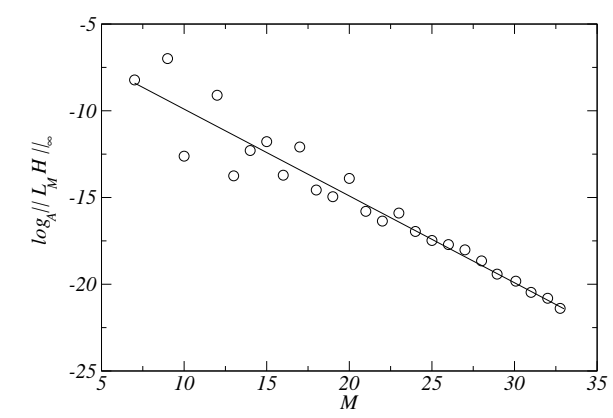

Figure 9. The function $M \mapsto \log _{A}\left\|\mathcal{L}_{M} \mathcal{H}\right\|_{\infty}$ and the linear fit $\log _{A}\left\|\mathcal{L}_{M} \mathcal{H}\right\|_{\infty}=C_{D L P}-\eta_{D L P} M$.

5.2. Conclusion. We conclude this paper by summarizing the obtained results. We introduced the 1/2-complex Bruno function and the Yoccoz function, both with an algorithm to evaluate them numerically. We studied the function $\mathcal{H}(z)=$ $-i \mathbf{B}(z)+\log U\left(e^{2 \pi i z}\right)$ defined on the upper Poincaré plane, and we conclude that it can be extended to its closure, with a trace $\eta$-Hölder continuous. Numerical results based on the Littlewood-Paley Theory give us the estimated value for the Hölder exponent: $\eta_{C L P}=0.498$ with an error of \pm 0.004 . We can then conclude, with a good numerical evidence, that the Marmi-Moussa-Yoccoz Conjecture should hold with the maximal exponent $1 / 2$.

\section{Appendix A. Numerical CONSIDERATIONS}

The aim of this Appendix is to consider in details some technical parts of our numerical calculations. We will consider the role of the cut-off and their relations with the accuracy of the computations. We will also compare the numerical properties of $\mathbf{B}$ with the analytical ones proved in Marmi et al. 2001.

\footnotetext{
${ }^{12}$ The exact value of $A$ is fixed in such a way we can take $M$ sufficiently large to have a good asymptotic, even if we have a finite number of Fourier coefficients.
} 
A.1. Accuracy of the algorithm for $\mathbf{B}(z)$. Let us recall the formula defining the 1/2-complex Bruno function:

$$
\mathbf{B}(z)=\sum_{n \in \mathbb{Z}}\left[\sum_{g \in \hat{\mathcal{M}}} L_{g}\left(1+L_{\sigma}\right)\right] \varphi_{1 / 2}(z-n),
$$

as already observed we need to introduce three cut-off to compute it: $N_{\max }, k_{1}$ and $k_{2}$. The first one determine the largest Farey Series involved, namely only fractions $p / q$ s.t. $p / q \in[0,1),(p, q)=1$ and $q \leq N_{\max }$ will be considered to compute B. The other two cut-off: $k_{1} \geq k_{2}>0$, are introduced to truncate the sum over $\mathbb{Z}$. Because the larger is $q$, the smaller is its contribution to $\mathbf{B}$, to gain CPU times we decide to truncate the sum over $\mathbb{Z}$ at $|n| \leq k_{1}$ if $q$ is "small", and to $|n| \leq k_{2}$ if $q$ is "large". Results showed in Section [5] are obtained with $N_{\max }=151, k_{1}=80$ and $k_{2}=20$.

In the rest of this paragraph we will study the dependence of the computed Bruno function on the cut-off. Let us fix two cut-off but one, call it generically $M$, and let us stress the dependence of $\mathbf{B}$ on it, by setting $\mathbf{B}_{M}(z)$. We are then interested in studying the relative error: $\epsilon_{\text {rel }}(z, M)=\left|\mathbf{B}_{M}(z)-\mathbf{B}(z)\right| /|\mathbf{B}(z)|$, where $z$ is fixed and $\mathbf{B}(z)$ is numerically computed with some fixed large cut-off: $N_{\max }=101, k_{1}=80$ and $k_{2}=20$. Or we can consider $\bar{\epsilon}_{r e l}(M)$ the mean value of $\epsilon_{\text {rel }}(z, M)$ for $\Re z \in[0,1 / 2]$ and some fixed value of $\Im z>0$. In Table A.1 we report values of $\log _{10} \bar{\epsilon}_{\text {rel }}(M)$, for $M \in\left\{N_{\max }, k_{1}, k_{2}\right\}$. Whereas in Figure 10 we show, $\log _{10} \epsilon_{\text {rel }}(z, M)$, for $M=N_{\text {max }}$ and $M=k_{1}$ and $z \in\{\sqrt{2}-1,2-\mathcal{G}\}$. Clearly the larger are the cut-off the more accurate are the results, but recall that large cutoff implies large CPU times; in particular the CPU times increases almost linearly w.r.t. $k_{1}$ and $k_{2}$, but quadratically w.r.t. $N_{\max }$.
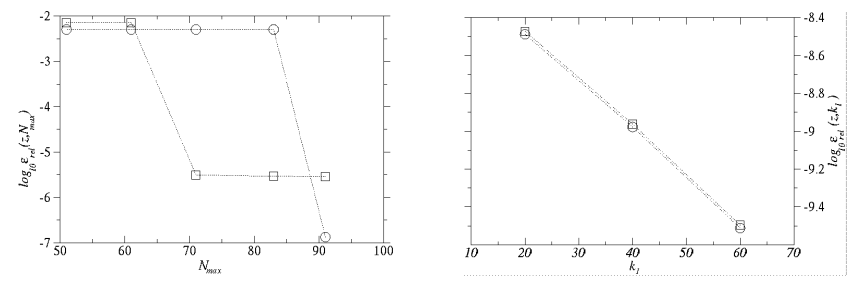

Figure 10. Plot of $\log _{10} \epsilon_{\text {rel }}(z, M)$ for some "good" $z$. On the left we show $\log _{10} \epsilon_{\text {rel }}\left(z, N_{\text {max }}\right)$ whereas on the right $\log _{10} \epsilon_{\text {rel }}\left(z, k_{1}\right)$. Circles are for $z=2-\mathcal{G}+i 10^{-7}$ and squares are for $z=\sqrt{2}-1+$ $i 10^{-7}$.

To have a full test of our algorithm we try to evaluate the limit, for $\Im z \rightarrow 0$, of the computed $\mathbf{B}$ and compare it with the results proved in Marmi et al. 2001]: section 5.2 .9 page 816 and Theorem 5.19 page 827 . In particular we will be interested in studying the rate of convergence of $\Im \mathbf{B}(x+i t)$ to $B_{1 / 2}(x)$, for $t \rightarrow 0$ when $x$ is some "good" number (Figure 11), and the "jump value" of $\Re \mathbf{B}(p / q+i t)$, when $t$ is "small" (Table A.2). 


\begin{tabular}{|c|c||c|c||c|c|}
\hline$N_{\text {qmax }}$ & $\log _{10} \bar{\epsilon}_{\text {rel }}\left(N_{\max }\right)$ & $k_{1}$ & $\log _{10} \bar{\epsilon}_{\text {rel }}\left(k_{1}\right)$ & $k_{2}$ & $\log _{10} \bar{\epsilon}_{r e l}\left(k_{2}\right)$ \\
\hline \hline 83 & -5.90 & 60 & -9.57 & 15 & -10.80 \\
61 & -4.73 & 40 & -9.10 & 10 & -10.32 \\
41 & -3.81 & 20 & -8.63 & & \\
\hline
\end{tabular}

TABLE 1. We report $\log _{10} \bar{\epsilon}_{r e l}\left(N_{\max }\right), \log _{10} \bar{\epsilon}_{r e l}\left(k_{1}\right)$ and $\log _{10} \bar{\epsilon}_{r e l}\left(k_{2}\right)$.

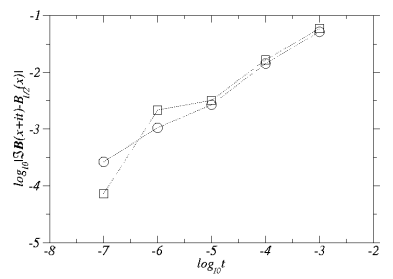

Figure 11. Convergence of $\Im \mathbf{B}(x+i t)$ to $B_{1 / 2}(x)$ when $t \rightarrow 0$ for some "good irrational' $x$. Circles are for $x=2-\mathcal{G}$ whereas squares for $x=\sqrt{2}-1$. We plot $\log _{10}\left|\Im \mathbf{B}(x+i t)-B_{1 / 2}(x)\right|$ versus $\log _{10} t$. $N_{\text {qmax }}=101, k_{1}=80$ and $k_{2}=20$.

\begin{tabular}{|c|c|}
\hline$p / q$ & $\Delta \Re \mathbf{B}(p / q+i t)-\pi / q$ \\
\hline \hline $0 / 1$ & $1.110^{-3}$ \\
$1 / 2$ & $7.410^{-4}$ \\
$1 / 3$ & $3.610^{-3}$ \\
$1 / 4$ & $3.610^{-3}$ \\
$1 / 5$ & $3.810^{-3}$ \\
$2 / 5$ & $2.510^{-3}$ \\
\hline
\end{tabular}

TABLE 2. The jumps of $\Re \mathbf{B}(x+i t)$ for rational $x$ and small $t$. The jump at $x=p / q$ is the numerical difference $\mid \Re \mathbf{B}(p / q+\delta+i t)-$ $\Re \mathbf{B}(p / q-\delta+i t) \mid$, for $\delta$ small. We report the difference of the jump w.r.t the expected value for $x \in\{0 / 1,1 / 2,1 / 3,1 / 4,1 / 5,2 / 5\}$ and $t=10^{-7}$.

\section{REFERENCES}

[Buff-Cheritat 2003] X. Buff and A. Cheritat: preprint at arXiv:math.DS/0305080 v1.

[Berretti Gentile 2001] A.Berretti and G.Gentile: Bryuno function and the standard map, Comm. Math. Phys., No. 4, 220, (2001), pp. 623-656.

[Buff et al. 2001] X. Buff, C. Henriksen and J. H. Hubbard: Farey curves, Experimental Mathematics, Vol. 10, No. 4, (2001), pp. 481-486.

[Buric et al. 1990] N. Buric, I. C. Percival and F. Vivaldi: Critical function and modular smoothing, Nonlinearity, 3, (1990), pp. 21-37.

[Carletti-Marmi 2000] T. Carletti and S. Marmi: Linearization of analytic and non-analytic germs of diffeomorphisms of $(\mathbb{C}, \mathbf{0})$, Bull. Soc. math. France, 128, (2000), pp. 69-85.

[Davie 1994] A.M. Davie: The critical function for the semistandard map, Nonlinearity, 7, (1994), pp. 219-229.

[Frazier et al. 1991] M. Frazier, B. Jawerth and G. Weiss:Littlewood-Paley Theory and the Study of Function Spaces, Conf. Board of the Math. Sciences, 79, AMS Providence, RI, 1991. 
[Hardy-Wright 1979] G.H. Hardy and E.M. Wright:An introduction to the theory of numbers, $5^{\text {th }}$ edition Oxford Univ. Press, 1979.

[Krantz 1983] S.G. Krantz:Lipschitz spaces, smoothness of functions, and approximation theory, Exposition. Math. 1, 3, (1983), pp. 193-260.

[Littlewood-Paley 1931] J.E. Littlewood and R.E.A.C. Paley: Theorems on Fourier series and power series, I, J. London Math. Soc., 6,(1931), pp. 230-233.

[De la Llave-Petrov 2002] R. de la Llave and N.P. Petrov: Regularity of conjugacies between critical circle maps: an experimental study, Experiment. Math., 11, 2, (2002), pp. 219-241.

[Marmi 1990] S. Marmi: Critical functions for complex analytic maps, J. Phys. A: Math. Gen. 23, (1990), pp. 3447-3474.

[Marmi et al. 1997] S. Marmi, P. Moussa and J.-C. Yoccoz: The Brjuno functions and their regularity properties, Communications in Mathematical Physics 186, (1997), pp. 265293.

[Marmi et al. 2001] S. Marmi, P. Moussa and J.-C. Yoccoz: Complex Brjuno functions, Journal of A.M.S. , Vol. 14, 4, (2001), pp. 783-841.

[Oesterlé 1993] J. Oesterlé: Polylogarithmes, Séminaire Bourbaki n. 762, Astérisque 216, (1993), pp. $49-67$.

[Stein 1970] E.M. Stein: Singular integrals and differentiability properties of functions, Princeton University Press (1970).

[Yoccoz 1995] J.-C. Yoccoz: Théorème de Siegel, polynômes quadratiques et nombres de Brjuno, Astérisque 231, (1995), pp. 3-88.

[Yoccoz 2002] J.-C. Yoccoz: Analytic linearization of circle diffeomorphisms, LNM, 1784, (2002), pp. $125-174$.

(Timoteo Carletti) Scuola Normale Superiore, piazza dei Cavalieri 7, 56126 Pisa, Italy

E-mail address, Timoteo Carletti: t.carletti@sns.it 